ELUA

ISSN 2171-6692

Núm. 36, 2021, págs. 27-44

https://doi.org/10.14198/ELUA.2021.36.02

\title{
Régimen y paráfrasis: sobre el tratamiento lexicográfico de las colocaciones verbonominales
}

\author{
Government and paraphrase: lexicographic treatment \\ of noun-verb collocations
}

Pablo Pérez Benavente

Universidad Complutense de Madrid, España

pperez12@ucm.es

https://orcid.org/0000-0003-1261-0853

\section{Resumen}

La Teoría Sentido-Texto (TST) integra las colocaciones en el diccionario mediante funciones léxicas, un lenguaje formal que recoge de forma sistemática este tipo de expresiones e informa, aunque solo de manera parcial, sobre su combinatoria. Esta información se incluye en todas las entradas lexicográficas, por lo que el uso de funciones léxicas puede entenderse como una consecuencia del estatuto ambiguo de las colocaciones, a medio camino entre el frasema y la combinatoria libre. En ocasiones es imposible predecir completamente la combinatoria sintáctica de una colocación más allá de lo incluido en la propia fórmula de la función léxica. En un diccionario que se pretende exhaustivo, como el empleado en la TST, esta información es importante no solo para un correcto procesamiento y producción de textos (el principal enfoque de la TST), sino también para la paráfrasis (otro de los puntos fuertes del modelo). El presente trabajo intenta
\end{abstract}

\begin{abstract}
The Meaning-Text Theory (MTT) has incorporated collocations in its special lexicographic model due to a mechanism called lexical functions, a formal language that systematically collects, in a particular section of a word's entry, all collocations whereby the semantic nucleus corresponds to this word. However, lexical functions do not provide information regarding a complete behaviour description of collocations. In fact, a complete description of this behaviour is included for all words with a lexicographic entry and therefore, lexical functions limited informativeness can be conceived as a consequence of the ambiguous status of collocations between full-lexical units and free-syntactic combinatory. The problem is that, frequently, their combinatorial behaviour cannot be predicted from regular combinatorial procedures. In a lexicographic work that attempts to describe the complete behaviour of a language, such as the one used in the MTT,
\end{abstract}

Para citar este artículo: Pérez Benavente, P. (2021). Régimen y paráfrasis: sobre el tratamiento lexicográfico de las colocaciones verbonominales. ELUA, (36), 27-44. https://doi.org/10.14198/ ELUA.2021.36.02

Recibido: 28/07/2021, Aceptado: 01/10/2021

(C) 2021 Pablo Pérez Benavente

Este trabajo está sujeto a una licencia de Reconocimiento 4.0 Internacional de

Creative Commons (CC BY 4.0) 
aportar diversas ideas a esta reflexión a partir de un conjunto de colocaciones verbonominales pertenecientes a dos campos semánticos de comportamiento dispar: el de los golpes y el de los sentimientos. En primer lugar, se ha realizado un análisis sintáctico-semántico de los regímenes de las colocaciones y sus verbos plenos equivalentes siguiendo el modelo lingüístico de la TST. A continuación, se han comparado ambas combinatorias con el objetivo de asegurar la máxima fidelidad en el proceso de sustitución por paráfrasis. Finalmente, se han recogido diversos instrumentos lexicográficos, coherentes con la estructura del diccionario empleado en la TST, que tratan de describir el comportamiento observado en el análisis, complementado con mecanismos que ponen en comunicación unas secciones del diccionario con otras. Estos dispositivos mixtos permitirían la caracterización precisa de las colocaciones sin que sea necesario el tratamiento particularizado de cada expresión como si se tratase de unidades léxicas plenas.

PALABRAS CLAVE: colocaciones; lexicografía; Teoría Sentido-Texto; régimen; paráfrasis. this information is fundamental not only for text production and processing (the main target of the MTT), but also for paraphrasing (the other strength of the model). The present paper aims to provide diverse ideas regarding this issue. In order to do this it works with a group of nounverb collocations belonging to two semantic fields with a very uneven behaviour: 'kicks' and 'feelings'. Following the linguistic model of the MTT, the rection of noun-verb collocations and that of their equivalent full-verb is syntactically and semantically analysed. Subsequently, in order to assure the highest fidelity in the paraphrasing process, both behaviours are compared. Finally, the work includes several possible lexicographic instruments, corresponding with the structure of the dictionary that intend to describe the particular data analysed. There are also suggested mechanisms that communicate different lexicographic sections. In combination, these mixed devices would allow a precise description of noun-verb collocations, without a particularized treatment of each term, as if they were full-lexical units.

KEYWORDS: collocations; lexicography; Meaning-Text Theory; rection; paraphrase.

\section{INTRODUCCIÓN}

\subsection{EI modelo Sentido-Texto}

El correcto procesamiento, la comprensión y la generación de textos son competencias fundamentales para el desarrollo de Inteligencia Artificial, y son varias las orientaciones lingüísticas nacidas en torno a esta idea. La Teoría Sentido-Texto (TST) (Zolklovskij y Mel'čuk 1965) concibe la lengua como un sistema en el que hablante y oyente elaboran mensajes físicos (textos) desde contenidos psíquicos (sentidos) y viceversa. La gramática sería un aparato lógico transformador capaz de generar un tipo de elementos a partir del otro (Alonso Ramos 1989), y aunque el proceso es bidireccional, la TST prima la síntesis, pues "la lengua es, ante todo, un medio de expresar significados" (Alonso Ramos 1998: 53). La correlación entre sentidos y textos es, en realidad, una correlación entre diversos niveles intermedios situados entre el fenómeno puramente físico y el puramente psíquico. La TST propone siete dimensiones lingüísticas, cada una con sus propias unidades y relaciones (Mel'čuk 1988), aunque para este trabajo bastará con conocer los tres niveles más próximos al elemento psíquico o sentido: la Estructura Semántica (SemS), la Estructura Sintáctica Profunda (DSyntS) y la Estructura Sintáctica Superficial (SSyntS). En adelante usaremos estas siglas para referirnos a los conceptos anteriores. 
Estos niveles se corresponden con las disciplinas lingüísticas tradicionales (semántica y sintaxis), y su tarea es describir las reglas de transformación que explican la transición de unos niveles a otros. Además, la TST también concede "gran importancia al léxico como alimento de todos los niveles y correspondencias" (Alonso Ramos 2004b: 30) y para ello emplea un lexicón particular llamado DEC (Diccionario Explicativo Combinatorio) que concibe la lexicografía como una recopilación de toda la información relevante para el uso de una palabra. En cada entrada se incluye una definición semántica, pero también una descripción de la combinatoria sintáctica y la combinatoria léxica. Un buen ejemplo de este tipo de diccionario puede comprobarse en el Dictionnaire explicatif et combinatoire du français contemporain. Recherches lexicosemantiques (Mel'čuk et alii 1984). Explicaremos en qué consiste cada una de las secciones de las que consta la entrada léxica en este tipo de diccionarios.

1) La definición semántica de un lexema es su forma proposicional. Todos los lexemas representan una situación que incluye determinados participantes, denominados actantes semánticos o argumentos, y estos aparecen en la forma proposicional por medio de las letras $\mathrm{X}, \mathrm{Y}, \mathrm{Z}, \mathrm{W}$, etc. El conjunto de argumentos de un lexema representa el esqueleto de su propia definición (Bosque y Gutiérrez-Rexach 2009).

2) La combinatoria sintáctica se representa por medio de un esquema de régimen (Government Pattern (GP) en inglés), una tabla que recoge la correspondencia entre los actantes semánticos del lexema y sus realizaciones sintácticas, o dicho de otro modo, entre su estructura semántica y su estructura sintáctica. La sintaxis que proyecta un elemento léxico (Hale y Keyser 1998) puede describirse, según nuestro modelo, de dos maneras en función de si se contempla desde el punto de vista del sentido o del texto. La Estructura Sintáctica Profunda (DSyntS), próxima al sentido, describe relaciones sintácticas lingüísticamente universales, abstractas, generales y muy productivas, que se corresponden con diferentes tipos de expresiones superficiales (Mel'čuk 2014). Estas se denominan Relaciones Sintácticas Profundas, y son proyectadas por un elemento léxico sobre otro que depende de él y que constituye su Actante Sintáctico Profundo (DSyntA). Los DSyntAs se identifican con números romanos del I al VI en un orden creciente de mayor oblicuidad respecto del lexema del que dependen (una jerarquía que se obtiene a partir del análisis de su comportamiento). Cada Relación Sintáctica Profunda se corresponde con una familia de Relaciones Sintácticas Superficiales (Mel'čuk 2004):

- I DSyntA $\rightarrow$ sujeto; complementos nominales de tipo parte-todo, continente-contenido, etc.

- II DSyntA $\rightarrow$ objeto directo; objeto indirecto en ausencia de objeto directo; complementos de preposiciones y conjunciones; complementos nominales que quedan fuera de DSyntA I; complemento agente.

- III DSyntA $\rightarrow$ objeto indirecto cuando coexiste con un objeto directo.

- IV - VI DSyntA $\rightarrow$ objetos preposicionales; objetos de mayor oblicuidad que los anteriores.

La Estructura Sintáctica Superficial (SSyntA) estaría más apegada al texto y reflejaría las relaciones sintácticas superficiales atendiendo a criterios formales. Aquí el orden lineal de las palabras, su carácter gramatical o su pertenencia a expresiones fraseológicas sí tienen reflejo en la representación sintáctica (Mel’čuk 2018). 
3) Por último, la combinatoria léxica remite al comportamiento combinatorio que no puede deducirse a partir de criterios semántico-sintácticos porque está motivado por cuestiones léxicas. Efectivamente, la combinatoria que funciona en las colocaciones es predominantemente de tipo léxico, puesto que el uso de una palabra no depende tanto de su significado como de "la preferencia, cuando se emplea una palabra, a utilizar otra determinada" (Koike 2001).

Si quiere expresarse la situación en la que se da un secreto, emplearemos el verbo contar, pero si de lo que quiere hablarse es de un discurso el verbo escogido será pronunciar. La palabra escogida por el hablante recibe el nombre de base, y aquella pre-seleccionada por la socialización recibe el nombre de colocativo (Írsula 1994). Este fenómeno se conoce como co-ocurrencia léxica restringida y sitúa las colocaciones a medio camino entre la combinatoria libre de palabras y la locución. La tercera parte de la entrada léxica se encarga de describir este tipo de comportamiento.

\subsection{Defectos de la descripción de las colocaciones en la TST}

El estatuto ambiguo de las colocaciones plantea todo un desafío para su tratamiento lexicográfico. En la TST, las unidades léxicas cuentan con una entrada propia en el diccionario, mientras que el análisis de las combinaciones libres es delegado a la aplicación de las reglas generales de la gramática. Las colocaciones, a medio camino entre unidad léxica y combinación libre, son recogidas en la sección de la combinatoria léxica de su palabra base. En la entrada de secreto se señalará que la colocación se forma con contar, mientras que en la de discurso se indicará su combinación con pronunciar.

La combinatoria léxica de una palabra se formaliza mediante un dispositivo conocido como Funciones Léxicas (FL) (Mel'čuk 2006). Las FL, inspiradas en funciones matemáticas, relacionan una unidad léxica $L$ con otra u otras unidades léxicas $L i$ a partir de determinada información general. La formulación de una FL genérica $\Lambda$ es:

$$
\kappa(L)=L_{i}
$$

donde $\Lambda$ es la identidad de la FL e indica la relación que existe entre $L$ y $L_{i}$; $L$ es la palabra a la que se aplica la FL, denominada palabra llave; y $L_{i}$ es aquella palabra o palabras que mantienen una relación de tipo $K$ con $L$, denominada/s valor de la FL.

Las FL son un dispositivo muy productivo a la hora de expresar relaciones entre palabras. Por ejemplo, la FL Anti relaciona una palabra con su antónimo, de modo que Anti $($ bueno $)=$ malo, Anti $($ arriba $)=$ abajo. Es una FL paradigmática, puesto que relaciona unidades léxicas incompatibles en un mismo sintagma. Por el contrario, las FL sintagmáticas relacionan palabras pertenecientes a distintas partes del discurso que sí pueden coexistir. La FL Magn relaciona una palabra con su cuantificador intensivo, de modo que Magn (hambre) $=$ atroz, Magn $($ error $)=$ garrafal. Dado que la colocación es una combinación recurrente de palabras, las FL que formalizan colocaciones son siempre sintagmáticas.

Una colocación verbonominal es una combinación de un verbo y un nombre en la que el nombre es escogido por el hablante (y constituye la base de la colocación), mientras que el verbo se ha estandarizado y depende del nombre (y constituye el colocativo). Las FL que expresan colocaciones siempre toman el sustantivo-base como palabra llave y dan como 
valor el verbo-colocativo. Las FL básicas para las colocaciones verbonominales son Oper , $_{\text {, }}$ Func $_{\mathrm{i}}$ y Labor $_{\mathrm{ij}}$. La relación que señalan es la misma: el verbo-valor de la FL forma una colocación con el sustantivo-palabra llave. Pero difieren en la correspondencia entre actantes semánticos y sintácticos:

- En el caso de Func $_{\mathbf{i}}$, el primer actante sintáctico (generalmente con función de sujeto) es el $i$ actante semántico del nombre.

- En el caso de $\mathbf{O p e r}_{\mathbf{i}}$, el segundo actante sintáctico (generalmente con función de objeto directo) es el $i$ actante semántico del nombre.

- En el caso de Labor ${ }_{\mathrm{ij}}$, el primer actante sintáctico es el $i$ actante semántico del nombre, y el segundo actante sintáctico es el $j$ actante semántico del nombre.

\begin{tabular}{|l|l|l|}
\hline \multicolumn{1}{|c|}{ UL } & \multicolumn{1}{|c|}{ Forma proposicional } & \multicolumn{1}{|c|}{ Ejemplos de FL } \\
\hline admiración & $\begin{array}{l}\text { 'admiración del individuo X al individuo } \\
\text { Y por el hecho Z' }\end{array}$ & $\begin{array}{l}\text { Oper }_{1}(\text { admiración })=\text { rendir }[\sim] \\
\mathbf{O p e r}_{2}(\text { admiración })=\text { gozar }[\text { de ART } ~]\end{array}$ \\
\hline odio & 'odio del individuo X al individuo Y por Z' & $\begin{array}{l}\text { Func }_{1}(\text { odio })=\operatorname{anidar}[\text { en X] } \\
\text { Func }_{2}(\text { odio })=\operatorname{dirigirse~}[\text { Prep Y] }\end{array}$ \\
\hline asco & 'asco del individuo X a Y' & Labor $_{12}($ asco $)=\operatorname{tratar~}[\mathrm{Y}$ con $\sim]$ \\
\hline
\end{tabular}

Tabla 1: Ejemplos de las FL colocacionales Oper, Func y Labor tomadas de DICE ${ }^{1}$

La FL más frecuente es Oper $_{i}$, que engloba expresiones como sentir admiración por alguien, gozar del respeto de alguien o constituir un desprecio a alguien. Sentir admiración equivale a Oper ${ }_{1}$ : el sujeto primer actante del sentimiento ('admiración de individuo $\mathrm{X}$ a individuo Y por hecho Z'). Gozar del respeto de alguien se corresponde con $\mathbf{O p e r}_{2}$ : el sujeto es el segundo actante del sentimiento ('respeto de individuo $\mathrm{X}$ hacia entidad $\mathrm{Y}$ por hecho Z'). Constituir un desprecio a alguien se corresponde con $\mathbf{O p e r}_{3}$ : el sujeto es el motivo y tercer actante del sentimiento ('desprecio de individuo X a Y consistente en $\mathrm{Z}$ ').

La información contenida en la fórmula de una FL (su identidad y subíndices) no detalla la realización sintáctica exacta, pero sí debe informar sobre la oblicuidad de los actantes "para facilitar la realización comunicativa" (Kahane y Polguère 2001). No obstante, mientras que la FL informa sobre un número fijo y limitado de posiciones sintácticas, el número de actantes puede variar de una colocación a otra ('admiración' de X a Y por Z; 'asco' de X a Y). La capacidad descriptiva de las fórmulas no es la misma en todas las colocaciones: si el nombre solo tiene un actante, la FL puede describir su estructura completa; pero abundan los nombres con dos o más actantes, inabarcables para una fórmula de FL (que como máximo nos informa de dos actantes). Las posiciones centrales son la de sujeto y la de objeto directo, generalmente reservadas para el propio nombre y uno de sus actantes semánticos, y son estas sobre las que nos informa la fórmula de una FL. Cuando el nombre (1) tiene más de un actante semántico ( 2 o más), el verbo deberá disponer de tres posiciones sintácticas, pero estas son impredecibles a partir de la FL. A continuación veremos varios casos.

Es cierto que algunas divergencias en la realización de los actantes son irrelevantes para la gestión de la información; un cambio sintáctico no tiene porqué alterar el número de

1 Ejemplos de FL y proposiciones semánticas tomados de DiCE (Alonso Ramos 2004b). 
sintagmas, ni tampoco su prominencia temática. En la expresión dar un golpe a la mesal en la mesa, el segundo actante del nombre se realiza de dos maneras, como objeto indirecto y como complemento circunstancial, pero uno y otro ocupan el mismo lugar en una oración prácticamente idéntica. Sin embargo, la variación tener miedo de la oscuridad/a la oscuridad sí acarrea cambios en la estructura informativa porque se da una variación en las relaciones de dependencia: el segundo actante semántico del nombre puede depender sintácticamente del propio nombre (miedo de la oscuridad) como II(N) DSyntA, formando un único constituyente; pero también puede depender del verbo como III DSyntA (tener(le) miedo a la oscuridad), dando lugar a dos piezas informativas independientes.

Lo que los ejemplos anteriores demuestran es que este comportamiento no puede reconstruirse solo mediante la fórmula de la FL. Y conocer el esqueleto sintáctico de una colocación es fundamental para la paráfrasis, otro de los principales objetivos de la TST. Las FL colocacionales (Oper1 (admiración) $=$ sentir $)$ pueden relacionarse fácilmente con la FL $\mathbf{V}_{\mathbf{0}}$, que da como valor verbos plenos semánticamente equivalentes a la colocación $\left(\mathbf{V}_{0}(\right.$ admiración $)=$ admirar $)$, un proceso cuyos detalles pueden comprobarse en Sanromán Vilas et alii (1999). Pero los ejemplos siguientes demuestran que esta sustitución puede acarrear importantes cambios en la estructura informativa. Por ejemplo, la información se organiza prácticamente de la misma manera en (1)a y en (1)b, pero no así en (2)a y (2)b, a pesar de que ambos pares se basan en una alternancia entre la colocación y el verbo pleno. Asimismo, (3)a es equivalente a (3)b en cuanto a número y orden de constituyentes, pero no está claro si la información de (4)a se conserva mejor en (4)b o en (4)c. Más adelante profundizaremos en estos casos.

(1) a. Mi hijo siente amor por la naturaleza.

b. Mi hijo ama la naturaleza.

(2) a. Los alumnos sienten alivio por haber terminado los exámenes.

b. Haber terminado los exámenes alivia a los alumnos.

(3) a. Juan golpeó las ventanas de la casa.

b. Juan dio un golpe a las ventanas de la casa.

(4) a. Juan golpeó la nariz a su primo.

b. Juan dio un golpe a la nariz de su primo.

c. Juan dio un golpe a su primo en la nariz.

En síntesis, aunque la formalización de las colocaciones mediante FL a partir del nombre facilita su paráfrasis, el funcionamiento del mecanismo aún debe mejorarse. Las FL constituyen una herramienta efectiva e intuitiva, pero en casos como (2) o (4) no permiten que se cumpla el principio de exhaustividad descriptiva que caracteriza a un diccionario tipo DEC. Es por ello que muchos diccionarios que trabajan con FL se ven forzados a informar sobre el esqueleto sintáctico de cada colocación en la entrada de la palabra llave. Así, Oper $($ asco $)=$ sentir $\left[\sim\right.$ por/de Y], mientras que Oper ${ }_{1}$ (asco $)=$ tener $[\sim$ a Y $]$ (ejemplo tomado de Alonso Ramos 2004b). Pero esta descripción particularizada de la colocación no hace sino contrarrestar la agilidad y sistematización descriptiva logradas gracias a las FL.

Con el objetivo de aportar algunas ideas en torno a esta problemática, analizaremos un conjunto de colocaciones extraídas del diccionario Diretes (Barrios Rodríguez 2020) y pertenecientes a dos campos semánticos: el de los golpes y el de los sentimientos. Todas ellas corresponden a la FL Oper, ${ }_{1}$, aunque su comportamiento es muy desigual. Las pertenecien- 
tes al campo semántico de los golpes son similares entre sí, por lo que es posible abarcar una gran cantidad de ejemplos (veintiséis sustantivos). Sin embargo, las pertenecientes al campo semántico de los sentimientos presentan gran variación en cuanto a verbos colocativos, estructura semántica y sintáctica, y presencia o ausencia de verbo equivalente. Por eso se ha seleccionado un subconjunto, basado en el orden alfabético y la frecuencia del nombre (trece sustantivos).

Desde el punto de vista metodológico, nuestro estudio comprende dos análisis diferentes. Primero, siguiendo el modelo lingüístico de la TST hemos descrito el esquema de régimen de cada uno de los conjuntos atendiendo a las variaciones que este presenta en función del nombre, el verbo o la combinación entre ambos. Los esquemas de régimen de este trabajo señalan la correspondencia entre actantes semánticos y actantes sintácticos profundos, sin atender a las diferentes realizaciones sintácticas superficiales. En otras palabras: solo se considera relevante la variación preposicional cuyas consecuencias sintácticas van más allá de la sustitución de una preposición por otra -como lo es, por ejemplo, el paso de un objeto indirecto a un complemento circunstancial. A continuación describimos el esquema de régimen de los verbos plenos equivalentes, vinculados a la colocación por medio del nombre, en los casos en los que existe este par en español. Tras comparar ambos esquemas de régimen, se propone una serie de elementos lexicográficos que integran dentro del diccionario los datos observados en el análisis, enriqueciendo la descripción de las colocaciones y, de este modo, también el mecanismo de la paráfrasis. Junto con la comunicación entre las diferentes secciones del diccionario, estos dispositivos mixtos pretenden aportar soluciones lexicográficas a la naturaleza híbrida de las colocaciones, evitando la descripción particularizada de cada una por separado.

\section{ESTUDIO DE UN CORPUS DE COLOCACIONES}

\subsection{Estudio de las colocaciones de golpe}

Las colocaciones estudiadas son del tipo dar un abanicazo, lanzar un balonazo, soltar una bofetada, pegar un cabezazo, propinar una cornada, etc. ${ }^{2}$.

\subsubsection{Esquema de régimen de las colocaciones de golpe}

Los verbos colocativos empleados en este tipo de construcciones son dar, lanzar, pegar y soltar (ocasionalmente enviar y tirar con balonazo; meter y propinar con cornada;

2 La lista completa de colocaciones pertenecientes al campo semántico de los golpes y su formalización es: Oper $_{1}($ abanicazo $)=$ dar, lanzar, pegar, soltar $; \mathbf{O p e r}_{1}($ balonazo $)=$ dar, enviar, lanzar, pegar, soltar, tirar; Oper $_{1}$ $($ bastonazo $)=$ dar, lanzar, pegar, soltar $; \mathbf{O p e r}_{1}($ bofetada $)=$ dar, lanzar, pegar, soltar $; \mathbf{O p e r}_{1}($ botellazo $)=$ dar, lanzar, pegar, soltar; Oper $_{1}($ cabezazo $)=$ dar, lanzar, pegar, soltar $;$ Oper $_{1}($ codazo $)=$ dar, lanzar, pegar, saltar; Oper $_{1}$ $($ coletazo $)=$ dar, lanzar, pegar, soltar $; \mathbf{O p e r}_{1}($ colleja $)=$ dar, lanzar, pegar, soltar $;$ Oper $_{1}($ cornada $)=$ dar, lanzar, meter, pegar, propinar, soltar; Oper $_{1}(\mathrm{coz})=$ dar, lanzar, pegar, soltar; $\mathbf{O p e r}_{1}($ derechazo $)=$ dar, lanzar, pegar, soltar $;$ Oper $_{1}($ espolada $)=$ dar, lanzar, pegar, soltar $; \mathbf{O p e r}_{1}($ gancho $)=$ dar, lanzar, pegar, soltar $; \mathbf{O p e r}_{1}($ golpe $)=$ atizar, dar, infligir, lanzar, pegar, propinar, soltar; $\mathbf{O p e r}_{1}($ martillazo $)=$ dar, lanzar, pegar, soltar; $\mathbf{O p e r}_{1}($ mazazo $)$ $=$ dar, lanzar, pegar, soltar $; \mathbf{O p e r}_{1}($ palo $)=$ dar, lanzar, pegar, soltar $; \mathbf{O p e r}_{1}($ patada $)=$ dar, lanzar, pegar, soltar $;$ Oper $_{1}($ pedrada $)=$ dar, lanzar, pegar, soltar $; \mathbf{O p e r}_{1}($ picotazo $)=$ dar, lanzar, pegar, soltar $; \mathbf{O p e r}_{1}($ planchazo $)=$ dar, lanzar, pegar, soltar; Oper $_{1}($ puñetazo $)=$ dar, lanzar, pegar soltar $; \mathbf{O p e r}_{1}($ rodillazo $)=$ dar, lanzar, pegar, soltar; Oper $_{1}($ torta $)=$ dar, lanzar, pegar, soltar $;$ Oper $_{1}($ zapatazo $)=$ dar, lanzar, pegar, soltar. 
y atizar, infligir y propinar con golpe) $)^{3}$. La estructura semántica de las colocaciones de golpe es siempre de tres actantes semánticos: X (quien golpea), Y (quien recibe el golpe) y Z (el lugar del golpe). Este último está incluido en el nombre de algunas colocaciones, como dar una colleja o soltar una bofetada (colleja y bofetada son golpes que incluyen en su propia definición las partes del cuerpo donde se recibe el golpe, los lugares de 'nuca' y 'mejilla'). Las restricciones semánticas son mínimas: X debe ser animado (humano en los casos de botellazo, gancho, martillazo, etc. y animal en coz, zarpazo, picotazo, etc.) y $\mathrm{Z}$ inanimado, pero no parecen existir restricciones sobre el tipo de entidad que puede funcionar como actante Y.

La Figura 1 recoge el esquema de régimen de las colocaciones de golpe, con la correspondencia entre sus actantes semánticos (fila superior) y sintácticos profundos (filas inferiores). Como corresponde siempre a la FL Oper $_{1}$, X $\rightarrow$ I DSyntA y N $\rightarrow$ II DSyntA. La realización sintáctica de los actantes $\mathrm{Y}$ y Z puede variar: pueden aparecer como complementos sintácticamente independientes, siendo Y $\rightarrow$ III DSyntA y Z $\rightarrow$ IV DSyntA, como se muestra en la fila central (Miguel le dio un codazo a su amigo en las costillas); o, como sintácticamente dependientes, siendo $\mathrm{Z} \rightarrow$ III DSyntA e $\mathrm{Y} \rightarrow \mathrm{II}(\mathrm{N})$ DSyntA, como se aprecia en la fila inferior (los manifestantes lanzaron pedradas a las ventanas del ayuntamiento). Esta posibilidad sintáctica se debe a la relación parte-todo que hay entre los actantes Y y Z, y provoca una "distancia emocional” (Sanromán Vilas 2009) que explica su preferencia para actantes $\mathrm{Y}$ no animados.

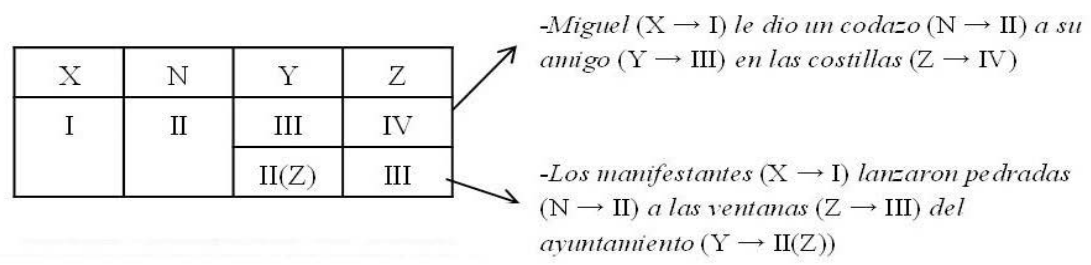

Figura 1: Esquema de régimen de las colocaciones pertenecientes al campo semántico de los golpes

\subsubsection{Colocaciones de golpe y su verbo pleno equivalente.}

Solo algunas colocaciones pertenecientes al campo semántico de los golpes presentan un verbo pleno semánticamente equivalente. Un vínculo morfológico no implica correspondencia semántica, pues de otro modo caeríamos en el error de equiparar dar un abanicazo con abanicar, dar un botellazo con embotellar o dar un codazo con codear(se). La Tabla 2 muestra los pares colocación-verbo pleno con un vínculo semántico que les permite aparecer en los mismos contextos.

3 La realización del diccionario Diretes aún está en curso, por lo que no cuenta con todas las colocaciones de muchos campos. Por ejemplo, podrían echarse en falta combinaciones como soltar un codazo o propinar una colleja. Aun así, consideramos que las generalizaciones que mencionaremos en este trabajo son extrapolables a otras colocaciones que no hemos analizado. 


\begin{tabular}{|l|l|}
\hline Colocaciones de golpe & Verbo pleno equivalente \\
\hline Oper $_{1}$ (bofetada) & Abofetear \\
\hline Oper $_{1}$ (cornada) & Cornear \\
\hline Oper $_{1}$ (coz) & Cocear \\
\hline Oper $_{1}$ (espolada) & Espolear \\
\hline Oper $_{1}$ (golpe) & Golpear \\
\hline Oper $_{1}$ (martillazo $)$ & Martillear \\
\hline Oper $_{1}$ (palo $)$ & Apalear \\
\hline Oper $_{1}$ (patada $)$ & Patear \\
\hline Oper $_{1}$ (pedrada $)$ & Apedrear \\
\hline Oper $_{1}$ (picotazo $)$ & Picar \\
& Picotear \\
\hline
\end{tabular}

Tabla 2: Colocaciones y verbos plenos equivalentes pertenecientes al campo semántico de los golpes

Los verbos plenos presentan los mismos actantes semánticos que las colocaciones (X, Y y Z) pero ahora es posible que X sea una entidad no animada: la crisis de 2008 golpeó duramente las arcas públicas (de Miguel 2006).

$\mathrm{Su}$ esquema de régimen, recogido en la Figura 2, muestra una diferencia lógica respecto a las colocaciones: en el caso de la colocación, es el objeto directo el que contiene el rasgo semántico de 'golpe'; sin embargo, el verbo pleno incluye en su propio significado ese sentido, por lo que ahora la posición de objeto directo está disponible para el resto de los participantes en la situación. Esto tiene importantes consecuencias sintácticas. Al disponer de la posición de objeto directo, las dos estructuras posibles en la colocación adquieren en el verbo pleno una menor oblicuidad respecto del verbo (como demuestran los números romanos más pequeños). Estas posibilidades están recogidas en las dos filas superiores con números romanos. La tercera fila, en la parte inferior, recoge una tercera realización sintáctica que carece de correlato colocacional: Y $\rightarrow$ III DSyntA, Z $\rightarrow$ II DSyntA (el viento golpeaba la cara a los excursionistas). Se trata de una combinación de las dos estructuras anteriores, que realiza Y y $\mathrm{Z}$ sintácticamente independientes pero sitúa $\mathrm{Z}$ en una posición más prominente.

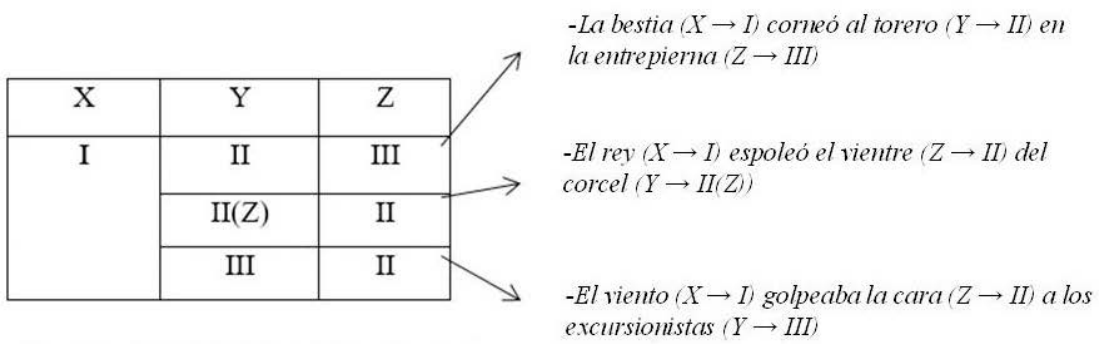

Figura 2: Esquema de régimen de los verbos plenos pertenecientes al campo semántico de los golpes 


\subsection{Propuestas para describir colocaciones de golpe}

\subsubsection{Comportamiento común a todo un conjunto}

Todas las colocaciones pertenecientes al campo semántico de los golpes presentan las mismas propiedades, para lo cual es útil emplear etiquetas semánticas. Este mecanismo reúne todo un conjunto bajo una etiqueta general y amplia con el fin de factorizar sus propiedades. El criterio del que la TST se vale para identificar estas etiquetas es el de la paráfrasis mínima, o posibilidad de sustituir en una expresión dada el nombre etiquetado por su etiqueta. Para una profundización en los detalles de este instrumento puede acudirse a Alonso Ramos (2004a: 160), quien sugiere emplear etiquetas específicas, que delimitan conjuntos tales como 'ruidos' o 'delitos'. Nuestro material exige una etiqueta semántica de 'golpe', como ya ha propuesto Barrios Rodríguez (2010: 217). La noción de 'golpe' constituye una parte central en la definición de estas expresiones, y el criterio de la paráfrasis mínima también apoya esta idea, como demuestra el uso de golpe como hiperónimo de pedrada y botellazo en los ejemplos de (5).

(5) Los niños dieron una pedrada a la ventana y el golpe rompió el cristal.

El botellazo que le dio fue el golpe que más le ha dolido en su vida.

Las etiquetas semánticas se integran en el DEC mediante una entrada léxica especial diseñada para este tipo de descripciones colectivas. La entrada léxica de la etiqueta 'golpe' incluiría los verbos colocativos compartidos, el esquema de régimen común y las restricciones semánticas asociadas a todas las colocaciones del grupo representando de una manera compacta gran parte de la co-ocurrencia léxica libre y restringida (Alonso Ramos 2004a). No obstante, esto implicaría cierta sobregeneralización ya que determinadas colocaciones nunca presentan de facto la estructura sintáctica asociada a la etiqueta. Por ejemplo, y como ya se ha mencionado, expresiones del tipo dar una colleja o una bofetada rara vez realizarán el actante $\mathrm{Z}$ o lugar del golpe ya que esta información viene incluida en su propio significado. Es necesario por tanto un cotejo entre la definición semántica del nombre y el esquema de régimen de la etiqueta que, una vez constatada la doble presencia de $\mathrm{Z}$, anule la posibilidad de realizar $Z$ y de hacer depender de él cualquier otro actante. El esquema de régimen de estas colocaciones quedaría como indica la Figura 3:

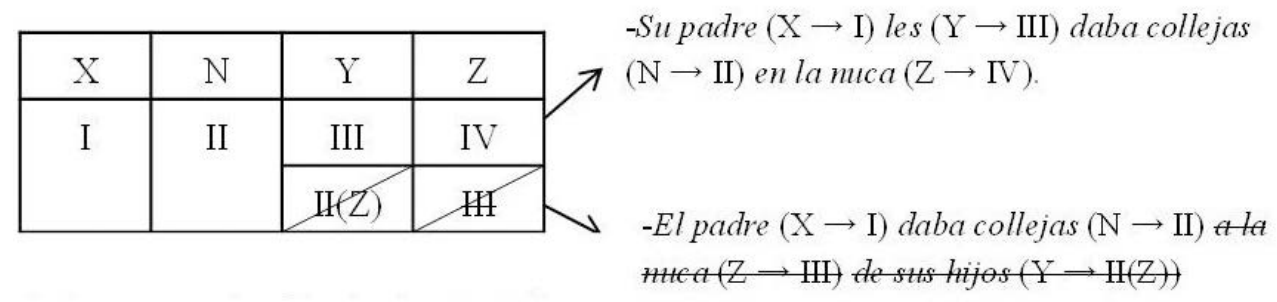

Figura 3: Esquema de régimen de una colocación que incluyera en su significado el actante $Z$ 


\subsubsection{Mantenimiento de la estructura informativa}

La Figura 4 reúne los esquemas de régimen de las colocaciones pertenecientes al campo semántico de los golpes y de los verbos equivalentes:

\begin{tabular}{|c|c|c|c|c|c|c|c|}
\hline $\mathrm{X}$ & $\mathrm{N}$ & $\mathrm{Y}$ & Z & \multirow{4}{*}{ 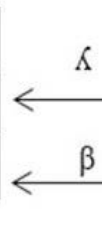 } & $\mathrm{X}$ & $\mathrm{Y}$ & Z \\
\hline \multirow[t]{2}{*}{ I } & \multirow[t]{2}{*}{ II } & III & IV & & \multirow[t]{3}{*}{ I } & II & III \\
\hline & & $\mathrm{II}(\mathrm{Z})$ & III & & & $\mathrm{II}(\mathrm{Z})$ & II \\
\hline & & & & & & III & II \\
\hline
\end{tabular}

Figura 4: Correspondencia entre los esquemas de régimen de colocaciones y verbos pleno del campo semántico de los golpes

Como hemos señalado, existe un claro paralelismo entre las dos primeras estructuras sintácticas de ambos esquemas de régimen. Para la transformación en cualquiera de las dos direcciones, una vez comprobada la compatibilidad semántica, proponemos etiquetar de forma distinta cada par de estructuras sintácticas ( $\kappa$ para la primera línea, $\beta$ para la segunda), asegurando una realización de tipo $\Lambda$ en la colocación cuando hay una estructura $\Lambda$ en el verbo pleno. Identificar y mantener las diferencias estructurales es fundamental para seleccionar la expresión que mejor se ajuste a la estructura comunicativa (Sanromán Vilas 1999).

La tercera realización del verbo, Y $\rightarrow$ III DSyntA y Z $\rightarrow$ II DSyntA, es una "combinación" de las otras dos estructuras: la prominencia de $Z$ (como $\beta$ ) y la independencia entre Y y Z (como $K$ ). Nunca será un punto de llegada desde la colocación, puesto que siempre será más oportuno emplear alguna de las otras dos estructuras disponibles. No obstante, sí debemos preguntarnos cuál es la mejor forma de trasladar esta expresión en sentido inverso: del verbo pleno a la colocación. En vez de establecer una correspondencia unívoca, lo mejor será valerse de otros mecanismos que detecten qué aspecto es más relevante: una mayor jerarquía de $\mathrm{Z}$ o una independencia sintáctica entre $\mathrm{Y}$ y $\mathrm{Z}$. Una vez evaluadas las consecuencias, puede entonces vincularse con una realización de tipo $\kappa$ o $\beta$.

\subsection{Estudio de las colocaciones de sentimiento}

Las colocaciones estudiadas son del tipo profesar admiración, sentir alivio, tener apego, cobrar asco, guardar rencor, etc. ${ }^{4}$.

4 El conjunto de colocaciones analizadas pertenecientes al campo semántico de los sentimientos y su formalización es: Oper ${ }_{1}($ admiración $)=$ profesar, sentir, tener; Oper $_{1}($ agobio $)=$ experimentar, padecer, sentir, soportar; Oper $_{1}($ alegria $)=$ experimentar, saborear, sentir, tener, vivir; Oper $_{1}($ alivio $)=$ experimentar, notar, sentir, tener; Oper $_{1}($ amor $)=$ albergar, experimentar, profesar, sentir, tener, vivir; $\mathbf{O p e r}_{1}($ apego $)=$ experimentar, profesar, sentir, tener; Oper $_{1}($ asco $)=$ albergar, cobrar, experimentar, hacer, pillar, profesar, sentir, soportar, tener; Oper $_{1}(c u l p a)$ $=$ tener, sentir $;$ Oper $_{1}($ deseo $)=$ acariciar, albergar, experimentar, sentir, tener $;$ Oper $_{1}($ estima $)=$ guardar, profesar, sentir, tener; Oper $_{1}($ miedo $)=$ albergar, experimentar, pasar, sentir, sobrellevar, soportar, tener; Oper $_{1}($ odio $)=$ albergar, encerrar, experimentar, guardar, notar, profesar, sentir, tener; $\mathbf{O p e r}_{1}($ rencor $)=$ abrigar, albergar, destilar, guardar, sentir, tener. 


\subsubsection{Esquema de régimen de las colocaciones de sentimiento}

Las colocaciones pertenecientes al campo semántico de los sentimientos son más variadas que las del conjunto anterior. La distribución de verbos colocativos en estas construcciones es muy desigual: sentir y tener se combinan con todos los sustantivos; experimentar, profesar y albergar se combinan con un buen número de ellos (nueve, seis y seis respectivamente); y el resto aparecen en el corpus una, dos o tres veces.

Se trata de construcciones con dos actantes semánticos, $\mathrm{X}$ e $\mathrm{Y}$, siendo $\mathrm{X}$ el experimentante del sentimiento e $\mathrm{Y}$ el origen u objeto del mismo. Las restricciones semánticas que imponen al primer actante es que $\mathrm{X}$ debe ser [+humano] pero el segundo, $\mathrm{Y}$, apenas tiene restricciones. No obstante, a diferencia de lo que ocurría con las colocaciones de 'golpes' no puede hablarse de una realización sintáctica prototípica. Por el contrario, los ejemplos analizados muestran una competencia entre dos estructuras sintácticas basadas, bien en una realización $\mathrm{Y} \rightarrow \mathrm{III}$ DSyntA del verbo, bien en $\mathrm{Y} \rightarrow \mathrm{II}(\mathrm{N})$ DSyntA. Las Figuras 5 y 6 ilustran estas dos estructuras. Mientras que solo unas pocas admiten una realización $\mathrm{Y} \rightarrow$ III DSyntA, todas ellas aceptan la construcción $\mathrm{Y} \rightarrow$ II.

\begin{tabular}{|c|c|c|}
\hline $\mathrm{X}$ & $\mathrm{N}$ & $\mathrm{Y}$ \\
\hline $\mathrm{I}$ & $\mathrm{II}$ & $\mathrm{II}(\mathrm{N})$ \\
\hline
\end{tabular}

$$
\begin{aligned}
& \text { - Carlos }(\mathrm{X} \rightarrow \mathrm{I}) \text { tiene miedo }(\mathrm{N} \rightarrow \mathrm{II}) \text { de } \\
& \text { suspender }(\mathrm{Y} \rightarrow \mathrm{I}(\mathrm{N})) \\
& \text { - Sentiamos apego }(\mathrm{N} \rightarrow \mathrm{II}) \text { por muestro } \\
& \text { barrio }(\mathrm{Y} \rightarrow \mathrm{II}(\mathrm{N})) \\
& \text {-Marta }(\mathrm{X} \rightarrow \mathrm{I}) \text { saborea la alegria }(\mathrm{N} \rightarrow \mathrm{II}) \\
& \text { del trabajo bien hecho }(\mathrm{Y} \rightarrow \mathrm{II}(\mathrm{N}))
\end{aligned}
$$

Figura 5: Esquema de régimen de una colocación que realiza $\mathrm{Y} \rightarrow \mathrm{II}(\mathrm{N}) \mathrm{DSyntA}$

\begin{tabular}{|c|c|c|}
\hline $\mathrm{X}$ & $\mathrm{N}$ & $\mathrm{Y}$ \\
\hline $\mathrm{I}$ & $\mathrm{II}$ & $\mathrm{II}(\mathrm{N})$ \\
\hline
\end{tabular}

Figura 6: Esquema de régimen de una colocación que realiza $\mathrm{Y} \rightarrow \mathrm{III}$ DSyntA

La posibilidad de encontrar una u otra estructura viene determinada por la combinación del nombre y el verbo. Nombres como alegría, alivio o culpa solo realizan $\mathrm{Y} \rightarrow \mathrm{II}(\mathrm{N})$ DSyntA. Otros como admiración admiten una realización de $\mathrm{Y} \rightarrow$ III DSyntA del verbo en función del verbo con el que se combinan, siempre y cuando se tomen en un sentido concreto 5 . Por último, hay casos, como miedo, en los que una construcción y otra son posibles con el mismo verbo.

5 Las construcciones admiración + verbo que permite $\mathrm{Y} \rightarrow$ III DSyntA solo son posibles cuando se toma admiración en un sentido próximo a 'respeto' o 'entusiasmo'. En un sentido próximo a 'asombro' o 'extrañeza', admiración se combina mayoritariamente con hechos (sentian profunda admiración ante todo lo que había en aquella habitación), no con personas, y es por lo tanto incompatible con construcciones del tipo Y $\rightarrow$ III DSyntA. 
Este comportamiento puede sistematizarse mejor si se analiza la combinación de verbos y nombres. Ya hemos visto que en la configuración de una colocación, el verbo es quien proporciona la estructura sintáctica, mientras que la estructura semántica es aportada por el sustantivo. Un verbo como sentir solo admite dos posiciones sintácticas (I siente II), y dado que estas están reservadas para el nombre (II) y uno de sus actantes (I), el actante restante deberá realizarse como dependiente del nombre por no disponer el verbo de más posiciones sintácticas. Mientras que uno de los actantes semánticos del nombre pasa sintácticamente al verbo, el otro se mantiene como dependiente del propio nombre:

i. $\quad$ I siente $\mathrm{II}+$ amor de $\mathrm{X}$ por $\mathrm{Y}=\mathrm{X}(\mathrm{I})$ siente amor por $\mathrm{Y}(\mathrm{II})$ 'alguien' siente 'algo' + amor de Carlos por los animales $=$ Carlos siente amor por los animales (lo siente).

Lo contrario sucede con verbos como profesar, que poseen tres posiciones sintácticas (I profesa II a III). Dispone de posiciones suficientes para el nombre y sus dos actantes, por lo que no es necesario que ninguno pase al nombre:

ii. I profesa II a III + admiración de $\mathrm{X}$ a $\mathrm{Y}=\mathrm{X}$ (I) profesa admiración(II) a Y(III) 'alguien' profesa 'algo' a 'alguien' + admiración de María a su profesor = María profesa admiración a su profesor (le profesa admiración).

En un punto intermedio se encuentran verbos como tener o albergar, con una estructura sintáctica variable (Alonso Ramos 2004a). Estos verbos pueden presentar ambas estructuras:

iii. I tiene II + apego de $\mathrm{X}$ por $\mathrm{Y}=\mathrm{X}(\mathrm{I})$ tiene apego por $\mathrm{Y}(\mathrm{II})$

'alguien' tiene 'algo' + apego de el niño por sus juguetes = el niño tiene apego por sus juguetes / lo tiene.

I le tiene II a III + apego de X a Y = X(I) le tiene apego(II) a Y(III)

'alguien' tiene 'algo' a 'alguien' + apego del niño a sus juguetes $=$ el niño tiene apego a sus juguetes / les tiene apego.

Organizando los 23 verbos que participan en estas colocaciones en función de si presentan una estructura sintáctica bivalente (i), trivalente (ii) o variable (iii), obtenemos la Tabla 3:

\begin{tabular}{|c|c|c|}
\hline $\begin{array}{l}\text { Verbos que realizan Y como } \\
\text { II DSyntA del nombre (i) }\end{array}$ & Verbos de realización variable (iii) & $\begin{array}{l}\text { Verbos que realizan Y como III } \\
\text { DSyntA del verbo (ii) }\end{array}$ \\
\hline $\begin{array}{l}\text { Sentir, experimentar, pade- } \\
\text { cer, soportar, sufrir, sabo- } \\
\text { rear, vivir, notar, soportar, } \\
\text { acariciar, pasar, sobrellevar, } \\
\text { abrigar }\end{array}$ & $\begin{array}{l}\text { Tener, albergar, destilar, ence- } \\
\text { rrar, guardar, hacer, pillar }\end{array}$ & Cobrar, profesar \\
\hline
\end{tabular}

Tabla 3: Verbos colocativos en función de la realización sintáctica de Y

Lo que determina la aparición de una estructura u otra es la combinación nombre-verbo, y es que la variación sintáctica asociada a los verbos de la columna central no es completa- 
mente libre, sino que depende del sustantivo con el que se combinen. Tras estudiar los datos hemos comprobado que solo con sustantivos que introducen Y mediante la preposición $a$ (apego a, admiración a, estima a, etc.) los verbos variables pueden desplegar una estructura trivalente o bivalente. Con el resto de los sustantivos (agobio por, deseo de, culpa por, etc.) solo presentarán una estructura bivalente. Asimismo, mientras que los verbos de la columna izquierda (bivalentes) pueden combinarse con todos los sustantivos, los verbos de la columna derecha (trivalentes) solo pueden combinarse con los verbos que introducen Y mediante la preposición a (*profesar alivio de, *cobrar agobio por, etc.).

\subsubsection{Colocaciones de sentimiento y su verbo pleno equivalente}

La mayoría de las colocaciones pertenecientes al campo semántico de los sentimientos presentan un verbo pleno que es morfológica y semánticamente equivalente. Las únicas que carecen de par verbal son Oper1 (apego), Oper1 (miedo) y Oper1 (rencor). No obstante, la equivalencia colocación-verbo no se da de la misma manera en todos los casos por las diferencias en la estructura sintáctica de los verbos, que alternan dos esquemas de régimen. Odiar, amar o estimar tienen una estructura $\mathrm{X} \rightarrow \mathrm{I}$ DSyntA, Y $\rightarrow$ II DSyntA, mientras que alegrar, agobiar o asquear tienen una estructura inversa $\mathrm{X} \rightarrow \mathrm{II}$ DSyntA, Y $\rightarrow$ I DSyntA. Estos dos grupos son los que Sanromán Vilas (2003) ha identificado como sentimientos de Causa Interna (CI) y sentimientos de Causa Externa (CE) respectivamente, y obedecerían a dos modos de concebir la realidad emocional. La Tabla 4 recoge la clasificación de los verbos plenos atendiendo a este criterio, y la Figura 7 indica sus respectivos esquemas de régimen ${ }^{6}$.

\begin{tabular}{|l|l|}
\hline \multicolumn{1}{|c|}{ Sentimientos de Causa Interna (CI) } & \multicolumn{1}{c|}{ Sentimientos de Causa Externa (CE) } \\
\hline Admirar & Agobiar \\
\hline Amar & Alegrar \\
\hline Desear & Aliviar \\
\hline Estimar & Asquear \\
\hline Odiar & \\
\hline
\end{tabular}

Tabla 4: Tipos de verbos plenos en función del origen del sentimiento

\begin{tabular}{|c|c|c|c|c|c|}
\hline \multicolumn{3}{|c|}{ Sentimientos de Causa Interna (CI) } & \multicolumn{3}{|c|}{ Sentimientos de Causa Externa (CE) } \\
\hline $\mathrm{x}$ & $\mathrm{Y}$ & \multirow{3}{*}{$\begin{array}{l}-Y o(\mathrm{X} \rightarrow \mathrm{I}) \text { admiro todo lo que } \\
\text { has hecho por mi }(\mathrm{Y} \rightarrow \mathrm{I}) \\
\text {-Es frecuente que los } \\
\text { adolescentes }(\mathrm{X} \rightarrow \mathrm{I}) \text { odien a sus } \\
\text { padres }(\mathrm{Y} \rightarrow \mathrm{II}) \text {. }\end{array}$} & $\mathrm{X}$ & $\mathrm{Y}$ & \multirow{3}{*}{$\begin{array}{l}- \text { La incertidumbre }(\mathrm{Y} \rightarrow \mathrm{I}) \\
\text { siempre agobia a quien la sufie } \\
(\mathrm{X} \rightarrow \mathrm{II}) \\
- \text { El sol de mavo }(\mathrm{Y} \rightarrow \mathrm{I}) \text { alegraba } \\
\text { los dias de mi infancia }(\mathrm{X} \rightarrow \mathrm{II})\end{array}$} \\
\hline I & II & & II & I & \\
\hline & & & & & \\
\hline
\end{tabular}

Figura 7: Esquema de régimen de los verbos plenos de sentimiento de Causa Interna (CI) y Causa Externa (CE)

6 Mención aparte merece el verbo culpar, cuyo I actante sintáctico no es ni X ni Y, sino un tercer actante Z. Esto se debe a que representa una situación que no es tanto el sentimiento de culpa como una adjudicación de la misma. 
Las restricciones semánticas que estos verbos imponen a sus actantes son las mismas que las colocaciones: $\mathrm{X}$ debe ser humano y no existen restricciones sobre Y. La excepción a la regla son los verbos alegrar y aliviar, que pueden llevar un $\mathrm{X}$ inanimado. Así sucede en el sol de mayo me alegraba los días $(\mathrm{X}=$ los días) o la noticia no alivió nuestro dolor $(\mathrm{X}$ = nuestro dolor). Este tipo de expresiones exigen que la entidad inanimada esté vinculada a un sujeto animado. Los ejemplos anteriores se aseguran de ello mediante un complemento indirecto (me alegra los dias) y un determinante posesivo (nuestro dolor).

\subsection{Propuestas para describir colocaciones de sentimiento}

\subsubsection{Variaciones asociadas a la combinación verbo-nombre}

Ya hemos visto que la mayor parte de los verbos son bivalentes y realizan $\mathrm{Y} \rightarrow \mathrm{II}(\mathrm{N})$ DSyntA. Un pequeño grupo de verbos, trivalentes, solo se combinan con nombres que introducen $\mathrm{Y}$ mediante la preposición $a$ y realizan $\mathrm{Y} \rightarrow$ III DSyntA del verbo. El tercer conjunto de verbos, de estructura sintáctica variable, podrán realizar Y $\rightarrow$ III DSyntA cuando el nombre con el que se combinan introduce $\mathrm{Y}$ mediante la $a$, y en el resto de los casos realizarán $\mathrm{Y}$ $\rightarrow \mathrm{II}(\mathrm{N})$ DSyntA.

Para recoger el comportamiento de estas colocaciones lo más apropiado es elaborar entradas de verbos colocativos. Análogas a las entradas de etiquetas semánticas, dan cuenta del comportamiento generalizable de ciertos conjuntos de expresiones. Esta entrada indica si el verbo en cuestión es bivalente, trivalente o de estructura variable. Los verbos de estructura variable deben tener acceso al régimen preposicional del sustantivo con el que se combinan, con objeto de comprobar si Y puede introducirse mediante $a$, ya que solo en esos casos será posible realizar $\mathrm{Y} \rightarrow$ III DSyntA, como se desprende del análisis de los datos. Para ello puede ser útil emplear la clasificación de sentimientos de Causa Interna y sentimientos de Causa Externa mencionada más arriba, ya que una de sus diferencias sintácticas es que los sentimientos de Causa Externa rechazan la introducción de Y mediante $a$. De este modo bloquearían una realización de $\mathrm{Y} \rightarrow$ III DSyntA al combinarse con verbos de estructura variable.

Seguir este criterio aseguraría la correcta generación de expresiones con verbos variables, pero también dinamizaría la introducción de nuevos verbos colocativos: en vez de introducirlos manualmente para cada sustantivo, bastaría con cotejar el régimen preposicional del nombre y el tipo de verbo (bivalente, trivalente o variable) para establecer las correspondencias de forma automática.

\subsubsection{Equivalencia semántica vs. Equivalencia informativa}

A diferencia de lo ocurrido en el campo semántico de los golpes, no existe una correspondencia entre las estructuras sintácticas de colocaciones y verbos plenos de sentimientos. Esto se debe a que entre los verbos plenos coexisten dos estructuras sintácticas opuestas.

$\mathrm{Al}$ sustituir un verbo por una colocación, lo más apropiado sería favorecer en lo posible realizaciones triactanciales, donde Y $\rightarrow$ III DSyntA (Jaime te estima $\rightarrow$ Jaime te tiene mucha estima). Así se consigue garantizar la independencia sintáctica que los actantes presentan con el verbo pleno. 
Para una paráfrasis inversa, de la colocación al verbo pleno, solo los verbos de sentimiento de Causa Interna tienen un reparto equivalente a la de la colocación, $X \rightarrow$ I DSyntA e $\mathrm{Y} \rightarrow$ II DSyntA. Para asegurar una paráfrasis lo más fiel posible conviene sustituir los verbos de sentimientos de Causa Externa (Y $\rightarrow$ I DSyntA y X $\rightarrow$ II DSyntA) por sus formas correspondientes incrementadas en -se (alegrarse, agobiarse, aliviarse). Estas formas invierten la distribución de los actantes, y son por lo tanto sintácticamente equivalentes a los verbos de sentimientos de Causa Interna y, por consiguiente, también a las colocaciones.

Tras estudiar los ejemplos creemos que esto puede realizarse por medio de la FL $\mathbf{V}_{\mathbf{0}}$, que aplicada a una palabra que no sea verbo selecciona como valor un verbo semánticamente equivalente a la palabra llave, cuyo sujeto (I DSyntA) es el actante X de la palabra llave. La clave radica en que, aplicada a sentimientos de Causa Interna, $\mathbf{V}_{\mathbf{0}}$ da verbos simples $\left(\mathbf{V}_{\mathbf{0}}\right.$ $($ amor $)=$ amar $)$, mientras que aplicada a sentimientos de Causa Externa da como valor verbos incrementados en -se $\left(\mathbf{V}_{\mathbf{0}}(\right.$ alivio $)=$ aliviarse $)$. Establecer la correspondencia bajo estos términos asegura una misma adjudicación de tema y rema a los participantes de la situación en ambas expresiones.

Por último, convendría identificar los casos en los que aliviar y alegrar aceptan un $\mathrm{X}$ inanimado, como los días o nuestro dolor, puesto que, aunque correctos, son imposibles de parafrasear por una colocación debido a la restricción que esta impone al actante X.

\section{CONCLUSIONES}

El estatuto ambiguo de las colocaciones, a medio camino entre la unidad léxica y la combinatoria libre, exige el desarrollo de mecanismos lexicográficos específicos para su integración y descripción en el diccionario. Las Funciones Léxicas juegan un importante papel en esta labor al recoger de manera sistemática las colocaciones en la entrada lexicográfica de la palabra base, pero la información sobre la combinatoria sintáctica contenida en la fórmula de la Función Léxica es insuficiente a la hora de predecir el comportamiento de ciertas expresiones. Si se persigue una caracterización completa, al tiempo que dinámica, es imprescindible desarrollar y profundizar mecanismos que eviten tener que describir cada colocación de forma individualizada.

Son varios los instrumentos que pueden servir a este fin. Para conjuntos de colocaciones con un comportamiento regular, emplear etiquetas semánticas que describan la combinatoria de todo un grupo de expresiones es lo más apropiado. Dado que la etiqueta siempre se corresponde con una parte central de la definición (la idea de 'golpe' es nuclear en palabras como pedrada), su activación puede automatizarse. También es importante que la entrada léxica de la etiqueta tenga acceso a la definición de la expresión para identificar qué informaciones se encuentran en el propio nombre y evitar así la redundancia que supondría realizarlas sintácticamente. Para conjuntos de colocaciones con un comportamiento más irregular, elaborar entradas léxicas de verbos colocativos es una alternativa al tratamiento pormenorizado de cada colocación. Estas entradas léxicas se activarían cuando los verbos aparecen como valor de una FL en la combinatoria léxica del sustantivo. Junto con el régimen preposicional del sustantivo, aportan la información necesaria para reconstruir el esqueleto sintáctico completo de cualquier colocación. Por ejemplo, tras activarse la colocación Oper1 (admiración) se activarían también las entradas de los verbos colocativos que participan en la colocación: profesar, sentir y tener. Profesar es un verbo trivalente, sentir 
es bivalente y tener es de estructura variable, como quedaría reflejado en su entrada como verbos colocativos. Además de su estructura actancial, estas entradas también deben recoger las consecuencias semánticas y sintácticas asociadas a cada construcción, a fin de disponer de la mayor cantidad de datos posible para acertar en el uso de uno u otro verbo.

El estudio de la combinatoria sintáctica de las colocaciones también es importante para asegurar una paráfrasis que mantenga la estructura temática. Sustituidas las descripciones esquemáticas incluidas en las FL por esquemas de régimen, se favorecería el mantenimiento de esquemas informativos si se etiquetaran de diferente manera las distintas estructuras atendiendo a criterios de oblicuidad y dependencia sintáctica. Una colocación como sentir alivio debe parafrasearse por la construcción equivalente con el verbo aliviarse, en vez de con aliviar.

Las propuestas contenidas en este trabajo no constituyen instrumentos definitivos para una caracterización de las colocaciones estudiadas. Para ello sería necesario ampliar el corpus de expresiones en el que se examine su validez, sobre todo en el caso de las colocaciones pertenecientes al campo semántico de los sentimientos. También habría que idear métodos para evitar sobregeneralizaciones y solapamientos con expresiones pertenecientes a otros ámbitos (el verbo tener también lo encontramos en otras colocaciones como tener una conversación, tener alergia, etc.). Con estas propuestas pretendemos anticipar alguno de los ejes centrales sobre los que puede sustentarse la descripción de expresiones léxicamente complejas o poliléxicas (Wotjak 1998). A nuestro juicio se ofrece como alternativa perfectamente viable a una descripción individualizada un enfoque que alternativamente delegue la tarea descriptiva en diferentes ámbitos de la construcción (el campo semántico, el verbo colocativo, el régimen preposicional, etc.) junto con una disponibilidad total entre las diferentes secciones de la entrada lexicográfica.

\section{REFERENCIAS BIBLIOGRÁFICAS}

Alonso Ramos, M. (1989). “Aproximación a un nuevo modelo lexicográfico: El Dictionnaire explicatif et combinatoire du français contemporain. Recherches lexico-sémantiques de Igor Mel'čuk". Verba: Anuario galego de filoloxia, 16, pp. 421-450

Alonso Ramos, M. (1998). "La tercera cara del signo lingüístico". Moenia: Revista lucense de lingüistica \& literatura, 3, pp. 51-77.

Alonso Ramos, M. (2004a). Las construcciones con verbo de apoyo. Madrid: Visor Libros.

Alonso Ramos, M. (2004b). DiCE Diccionario de colocaciones del español. Recuperado de http://www.dicesp.com/paginas.

Barrios Rodríguez, M. A. (2010). "El dominio de las funciones léxicas en el marco de la Teoría Sentido-Texto". Estudios de Lingüística del Español (ELiEs), 30.

Barrios Rodríguez, M. A. (2020). "The making of the Diretes dictionary". En Gavriilidou, Z., M. Mitsiaki y A. Fliatouras (eds.). Lexicography for inclusion. EURALEX Proceedings. XIX Congress of the European Association for Lexicography. Alexandroupolis: Democritus University of Thrace/ European Association for Lexicography, pp.13-22.

Barrios Rodríguez, M. A. y Rello, L. (2011). "False Paraphrase Pairs in Spanish for Verbs and Verb+Noun Collocations". Procesamiento del Lenguaje Natural, 46, pp. 107-112.

Bosque, I. y Gutiérrez-Rexach, J. (2009). "La predicación generalizada". En Fundamentos de sintaxis formal. Madrid: Akal, pp. 260-270.

Hale, K. y Keyser, S. J. (1998). "The Basic Elements of Argument Structure“. En Prolegomenon to a Theory of Argument Structure. Massachusets: MIT Press, pp. 1-47. 
Írsula, J. (1994). "Entre el verbo y el sustantivo, ¿quién rige a quién? El verbo en las colocaciones sustantivo-verbales', en Annette Endruschat et al. (coords.). Verbo e estruturas frásicas. Actas do IV Colóquio Internacional de Linguistica Hispânica de Leipzig. Oporto: Universidad de Oporto, pp. 277-286.

Kahane, S. y Polguère, A. (2001). "Formal foundation of lexical functions". En COLLOCATION: Computational Extraction, Analysis and Explitatition, Touluse, pp. 8 -15

Koike, K. (2001). Colocaciones léxicas en el español actual: estudio formal y léxico-semántico. Alcalá de Henares: Servicio de publicaciones de la Universidad de Alcalá.

Mel'čuk, I. (1988). Dependency Syntax: Theory and Practice. State University of New York Press.

Mel'čuk, I. (2004). "Actants in Semantics and Syntax. II. Actants in Syntax". Linguistics, 42(2), pp. 247-291. https://doi.org/10.1515/ling.2004.009

Mel'čuk, I. (2006). "Lexical Functions: A tool for the Description of Lexical Relations in a Lexicon". En Wanner, L. (ed.), Lexical functions in lexicography and natural Language processing, pp. 37-102.

Mel'čuk, I. (2014). "Dependency in Language“. En Gerdes, K., Hajicová, E. y Wanner, L. (eds.), Dependency Linguistics: Recent advances in linguistic theory using Dependency structures, pp. 1-32. https://doi.org/10.1075/la.215.01mel

Mel'čuk, I. (2018). "Anna Wierzbicka, Semantic Decomposition, and the Meaning-Text Approach". Russian Journal of Linguistics, 22(3), pp. 521-538. https://doi.org/10.22363/2312-9182-2018-223-521-538

Mel‘čuk, I., Arbatchewsky-Jumarie, N., Sagenais, L., Elnitsky, L., Iordankaja, L., Lefebvre, M. N., y Mantha, S. (1984). Dictionnaire explicatif et combinatoire du français contemporain. Recherches léxico-semántiques I, Montreál: Les Presses de l’Université de Montreál.

De Miguel, E. (2006). "Tensión y equilibrio semántico entre nombres y verbos: el reparto de la tarea de predicar". En Villayandre, M. (ed.) Actas del XXXV Simposio Internacional de la Sociedad Española de Lingüistica, León: Universidad de León, pp. 1289-1313. http://www3.unileon.es/dp/ $\mathrm{dfh} / \mathrm{SEL} / \mathrm{actas} . \mathrm{htm}$

Sanromán Vilas, B. (2003). Semántica, sintaxis y combinatoria léxica de los nombres de emoción en español (Tesis doctoral). Helsinki: Universidad de Helsinki.

Sanromán Vilas, B. (2009). "Diferencias semánticas entre construcciones con verbo de apoyo y sus correlatos verbales simples". Estudios de Lingüística Universidad de Alicante, 23, pp. 289-314. https://doi.org/10.14198/ELUA2009.23.13

Sanromán Vilas, B., Lareo Martín, I. y Alonso Ramos, M. (1999). “Transferencia léxica y reglas de paráfrasis: verbos denominales de SP cognado". Procesamiento del Lenguaje Natural, 25, pp. 183-189.

Zolkovskij, A. y Mel'čuk, I. (1965). "On a posible method an instrument for semantic synthesis of texts". Scientific and Technological Information, 6, pp. 23-28.

Wotjak, G. (1998). "Reflexiones acerca del potencial combinatorio sintagmático de las unidades léxicas / UL". Boletín de filología, 37(2), pp. 1284-1308. 